\title{
Surgical treatment of childhood hepatoblastoma in the Netherlands (1990-2013)
}

\author{
Linde A. D. Busweiler ${ }^{1}$ Marc H. W. A. Wijnen ${ }^{1,6} \cdot$ Jim C. H. Wilde ${ }^{1,7}$ • \\ Egbert Sieders $^{2}$ - Sheila E. J. Terwisscha van Scheltinga ${ }^{3}$ - L. W. Ernest van Heurn ${ }^{4}$. \\ Joseph Ziros ${ }^{5}$ Roel Bakx ${ }^{1} \cdot$ Hugo. A. Heij ${ }^{1}$
}

Accepted: 5 October 2016

(c) Springer-Verlag Berlin Heidelberg 2016

\begin{abstract}
Background Achievement of complete surgical resection plays a key role in the successful treatment of children with hepatoblastoma. The aim of this study is to assess the surgical outcomes after partial liver resections for hepatoblastoma, focusing on postoperative complications, resection margins, 30-day mortality, and long-term survival. Method Chart reviews were carried out on all patients treated for hepatoblastoma in the Netherlands between 1990 and 2013.

Results A total of 103 patients were included, of whom 94 underwent surgery. Partial hepatectomy was performed in 76 patients and 18 patients received a liver transplant as a primary procedure. In 42 of $73(58 \%)$ patients, one or more complications were reported. In 3 patients,
\end{abstract}

Linde A. D. Busweiler

l.a.d.busweiler@1umc.nl

1 Pediatric Surgical Center of Amsterdam, Emma Children's Hospital-Academic Medical Center and VU Medical Center, P.O. Box 22660, 1100 DD Amsterdam, The Netherlands

2 Department of Surgery, University Medical Center Groningen, Groningen, The Netherlands

3 Department of Pediatric Surgery, Sophia Children's HospitalErasmus Medical Center, Rotterdam, The Netherlands

4 Department of Pediatric Surgery, Maastricht University Medical Center, Maastricht, The Netherlands

5 Department of Pediatric Oncology, Emma Children's Hospital-Academic Medical Center, Amsterdam, The Netherlands

6 Department of Pediatric Surgery, Radboud University Medical Center, Nijmegen, The Netherlands

7 Present Address: Department of Pediatric Surgery, University Hospital, Geneva, Switzerland information regarding complications was not available. Hemorrhage necessitating blood transfusion occurred in 33 (45\%) patients and $9(12 \%)$ patients developed biliary complications, of whom 8 needed one or more additional surgical interventions. Overall, 5-year disease-specific survival was $82,92 \%$ in the group of patients who underwent partial hepatectomy, and $77 \%$ in the group of patients who underwent liver transplantation.

Conclusions Partial hepatectomy after chemotherapy in children with hepatoblastoma offers good chances of survival. This type of major surgery is associated with a high rate of surgical complications (58\%), which is not detrimental to survival.

Keywords Hepatoblastoma - Partial hepatectomy · Postoperative complications - Hemorrhage - Biliary complications

\section{Introduction}

Hepatoblastoma is the most common malignant liver tumor and the third most common intra-abdominal neoplasm in children in Western countries. Overall, survival has significantly improved from 35 to $83 \%$ over the last four decades due to advances in chemotherapy [1-5]. Complete surgical resection remains essential in the successful treatment for children with hepatoblastoma.

In adults, partial liver resections are associated with high complication rates, but in children, little is known about this topic [6]. Morbidity rates between 22 and $49 \%$ have been shown in literature and potential complications include hemorrhage, biliary leakage or obstruction, infection, liver failure, and tumor positive resection margins $[1,7-13]$. A better insight into the surgical outcomes and 
complications of partial liver resections could improve outcome and reduce morbidity.

The aim of this study was to give an overview of the experience with the surgical treatment of children with hepatoblastoma in the Netherlands between 1990 and 2013. This study focuses on the (surgical) treatment of the primary liver tumor. However, since the occurrence and management of distant metastases play an important role in the treatment for children with hepatoblastoma, this subject will be addressed in a less extensive manner.

\section{Methods}

\section{Patients}

A retrospective chart review was performed on all children treated for hepatoblastoma in one of the Pediatric Surgical Oncology Centers in the Netherlands between 1990 and 2013. These hospitals were the only national tertiary centers responsible for the treatment of hepatoblastoma in patients under the age of 18 years. Patient charts and surgical reports were used to collect information, regarding demographics, tumor characteristics, diagnostic tests, surgical treatment, postoperative morbidity (in the case of partial liver resection), mortality, and follow-up. Children were excluded from analysis if the histopathology report after resection or transplantation resulted in a diagnosis other than hepatoblastoma.

\section{Investigation methods}

Ultrasonography, chest radiography, and alpha-fetoprotein $(\alpha \mathrm{FP})$ levels in the blood were the first diagnostic tools used in all children with a suspicious abdominal mass. The diagnosis was made according to the International Childhood Liver Tumors Strategy Group (SIOPEL) protocols running at that time (SIOPEL 1-4) [1, 4, 14-16]. This included a pre-operative tumor biopsy according to SIOPEL 4, which was not mandatory for children between 6 months and 3 years with a solid hepatic mass and elevated serum $\alpha \mathrm{FP}$ in SIOPEL 1-3. However, following the results of a Dutch study published in 2005 , this was already promoted as a part of standard care [17].

Before starting with neoadjuvant chemotherapy, a computed tomography (CT) or Magnetic Resonance Imaging (MRI) scan was performed to evaluate tumor extension and involvement of vessels and other intra-abdominal organs. The PRETEXT system (PRE Treatment EXTent of disease staging system) was used for staging and risk stratification [18]. In addition, a CT scan of the chest was made to detect possible pulmonary metastases.
Tumor response and resectability after neoadjuvant chemotherapy were evaluated using either CT scan or MRI.

\section{Pre-operative treatment}

SIOPEL guidelines for the treatment of liver tumors in children were introduced in the Netherlands in the early 1990s. Since then, chemotherapy regimen consisted of a combination of cisplatin, doxorubicin, and carboplatin, according to SIOPEL 1-4 studies [1, 4, 14-16]. Before the introduction of these guidelines, patients received chemotherapy according to locally developed protocols.

\section{Surgical treatment}

\section{Primary tumor}

Patients were treated in one of the pediatric surgical centers in the Netherlands experienced in liver resections after treatment with neoadjuvant chemotherapy. Surgical treatment strategies were based on SIOPEL 1-4 protocols running at the time of diagnosis [1, 4, 14-16]. Standard surgical techniques for tumor resection were used [19]. During surgery, ultrasonography was used when considered necessary. If the primary tumor was considered unresectable after neoadjuvant chemotherapy, primary orthotopic liver transplantation was performed. Liver transplantation was indicated in the case of extensive (multifocal) disease (PRETEXT IV) or in the case of an unifocal centrally located tumor involving the main hilar structures or the main hepatic veins (SIOPEL 1-4) [20]. Transplantation was performed in a specialized transplant center. The vast majority of these transplantations were performed abroad.

\section{Metastatic disease}

If pulmonary metastases were still present after treatment with chemotherapy, metastasectomy [via sternotomy, thoracotomy or video assisted thoracoscopic surgery (VATS)] was performed whenever possible. This procedure was usually performed after complete removal of the primary liver tumor, except in candidates for liver transplant, who had to be free from metastases before liver surgery.

\section{Postoperative complications}

Information regarding postoperative complications after partial hepatectomy was used for analysis. Complications were defined as any deviation from the normal postoperative course. The following definitions were used:

Hemorrhage Perioperative bleeding necessitating blood transfusion or re-operation. 
Infection Any type of infection related to the surgical procedure necessitating the use of antibiotics or drainage (open or percutaneous) of abscesses.

Biliary complications Bile leak and/or a biliary tract stricture.

Vascular complications Compromised hepatic blood flow due to the surgical procedure.

Other Any other postoperative complications related to the surgical procedure requiring re-intervention and/or prolonged hospital stay.

\section{Follow-up}

All patients were followed-up at children's oncology outpatient clinics.

$\alpha \mathrm{FP}$ levels were determined and chest $\mathrm{X}$-rays and abdominal ultrasonography were performed to monitor recurrence according to SIOPEL-protocols.

\section{Statistical analysis}

Patient, tumor, and treatment characteristics were described using frequency tables. Disease-specific survival was calculated from the date of diagnosis until date of death (related to hepatoblastoma) or last follow-up. Patients who died due to other causes (not related to hepatoblastoma) were censored. Disease-specific survival was analyzed using the Kaplan-Meier survival analysis with a $95 \%$ confidence interval (CI). Median follow-up time was analyzed using a reverse Kaplan-Meier method. A $p$ value $<0.05$ was considered statistically significant. Statistical analyses were performed in the PASW Statistics version 21 (SPSS inc., Chicago, IL, USA).

\section{Results}

\section{Clinical features}

Between 1990 and 2013, a total of 103 children were treated for hepatoblastoma in the Netherlands. Patient and tumor characteristics are shown in Table 1.

\section{Surgical treatment}

\section{Primary tumor}

Nine $(9 \%)$ patients died before surgery was performed, due to progressive disease under therapy. Eighteen (17\%) patients underwent total hepatectomy combined with orthotopic liver transplantation as a primary procedure and $76(74 \%)$ patients underwent a partial liver resection (Fig. 1). Table 2 gives an overview of the different surgical
Table 1 Patient and tumor characteristics

\begin{tabular}{|c|c|c|}
\hline Category & Number & $(\%)$ \\
\hline \multicolumn{3}{|l|}{ Gender } \\
\hline Male & 62 & 60 \\
\hline Female & 41 & 40 \\
\hline \multicolumn{3}{|l|}{ Age (months) } \\
\hline Median & 15 & - \\
\hline Range & $0-162$ & - \\
\hline \multicolumn{3}{|l|}{$\mathrm{AFP}(\mu \mathrm{g})$} \\
\hline Mean & 432.614 & \\
\hline Median & 210.000 & \\
\hline Range & $15-380.400 .0$ & \\
\hline \multicolumn{3}{|l|}{ PRETEXT } \\
\hline I & 4 & 3.9 \\
\hline II & 25 & 24 \\
\hline III & 26 & 25 \\
\hline IV & 20 & 19 \\
\hline Missing & 28 & 27 \\
\hline \multicolumn{3}{|l|}{ Tumor location } \\
\hline Right & 68 & 66 \\
\hline Left & 8 & 8 \\
\hline Bilateral & 20 & 19 \\
\hline Unknown & 7 & 7 \\
\hline \multicolumn{3}{|l|}{ Histopathology } \\
\hline Epithelial & 30 & 29 \\
\hline Epithelial/mesenchymal & 39 & 38 \\
\hline Mesenchymal & 1 & 0.9 \\
\hline Not specified & 33 & 32 \\
\hline Local disease & 75 & 73 \\
\hline Metastatic disease & 28 & 27 \\
\hline Pulmonary & 27 & \\
\hline Pulmonary, spleen, pancreas & 1 & \\
\hline \multicolumn{3}{|l|}{ Type of liver tumor } \\
\hline Unifocal & 80 & 78 \\
\hline Multifocal & 23 & 22 \\
\hline \multicolumn{3}{|l|}{ Follow up (months) } \\
\hline Median & 61 & - \\
\hline Range & $0-213$ & - \\
\hline
\end{tabular}

PRETEXT pretreatment extent of disease

procedures performed. Thirty-four $(45 \%)$ patients underwent an extended hemihepatectomy, while in $10(13 \%)$ patients, partial liver resection was combined with several additional procedures.

\section{Metastatic disease}

Figure 2 shows the 28 patients diagnosed with metastatic disease (synchronous and metachronous). Seven of the twenty-two patients eligible for surgery with curative intent 


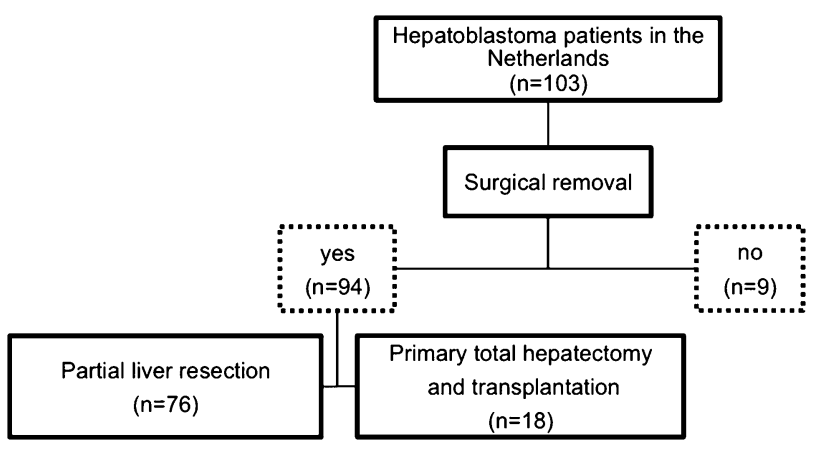

Fig. 1 Overview of the surgical treatment of patients with hepatoblastoma in the Netherlands (1990-2013)

had surgical resection of lung metastasis at varying times in their treatment. In three patients, metastasectomy was performed after surgical treatment of the primary liver tumor, and in three patients, this took place before primary tumor removal. Two of the latter patients eventually underwent total hepatectomy and primary liver transplantation. The third patient, initially planned for primary liver transplantation, responded well to chemotherapy and partial liver resection was performed successfully. In one patient, no information about metastasectomy was available. No metastases were resected simultaneously with liver resection.

\section{Surgical outcomes after partial liver resection}

\section{Resection margins}

Of 76 patients who underwent partial liver resection, the pathology report showed complete tumor removal in 66 $(87 \%)$ patients and microscopic tumor residue in $2(3 \%)$ patients. In $5(7 \%)$ patients, results were inconclusive which was followed by expectative treatment. In $3(4 \%)$ patients, there was no information about resection margins. None of the 10 patients with microscopic tumor residue or
Table 2 Overview of surgical procedures primary tumor

\begin{tabular}{lll}
\hline & Number & $\%$ \\
\hline Primary liver transplantation & 18 & 81 \\
Partial liver resection & 76 & 10 \\
Segmentectomy & 12 & 19 \\
Hemihepatectomy, left sided & 2 \\
Hemihepatectomy, right sided & 32 \\
Extended hemihepatectomy, left sided & 1 \\
Extended hemihepatectomy, right sided & 10 \\
Other, removal of pedunculated tumor & 1 \\
Partial liver resection combined with & 1 \\
Partial resection diaphragm & 1 \\
Partial resection diaphragm + cecal pole resection & 1 \\
Reconstruction inferior vena cava and left hepatic vein & 3 \\
Right sided adrenalectomy + additional segmentectomy & 2 \\
Additional segmentectomy & 1 \\
Resection portal vein & Roux-en-Y hepaticojejunostomy &
\end{tabular}

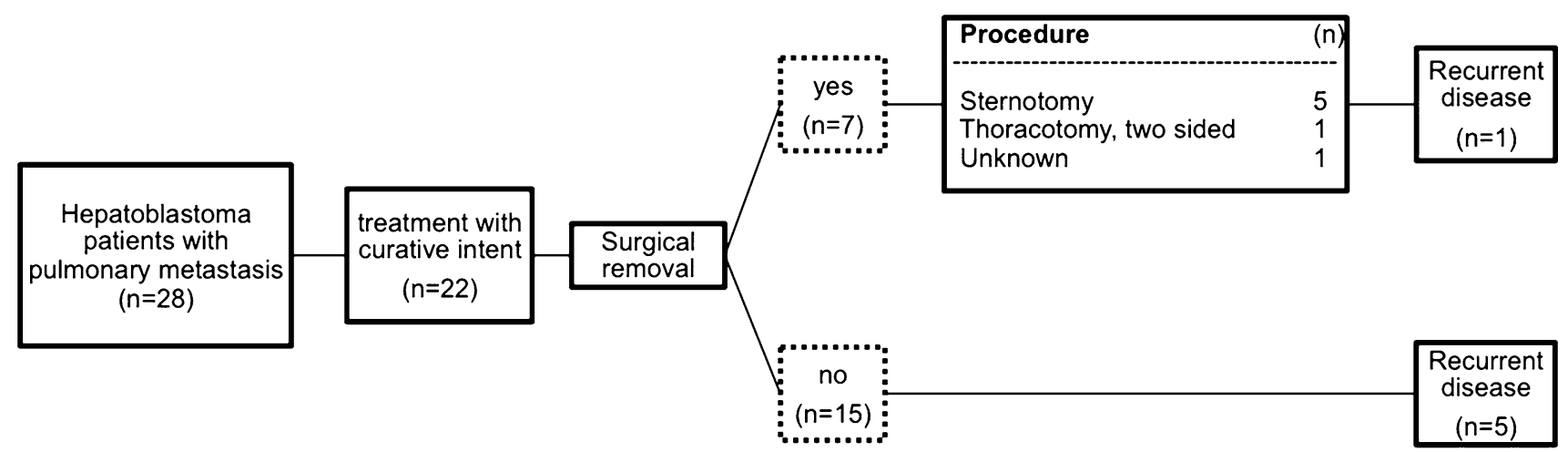

Fig. 2 Overview of the surgical treatment of metastatic disease in patients with hepatoblastoma in the Netherlands (1990-2013) 
missing or inconclusive data about resection margins presented with recurrent disease during follow-up.

\section{Postoperative complications}

Three patients without information about complications were, therefore, excluded from further analysis. In 42 $(58 \%)$ of 73 patients, one or more complications were reported. In total, 52 complications were registered (Table 3). Among the patients who experienced a complication, $43 \%$ were staged PRETEXT III or higher, compared to $32 \%$ in the group without a complication $(p=0.535)$ (Table 4).

Hemorrhage Thirty-three (45\%) patients received a blood transfusion because of perioperative hemorrhage. None of them required additional surgery. However, one of these patients (a 15-month-old infant with $1800 \mathrm{cc}$ blood loss) needed cardiopulmonary resuscitation (CPR) due to hemodynamic instability on the second day after surgery.

Infection In $6(8 \%)$ patients, a postoperative infection was reported, including two central venous line (CVL) infections/sepsis leading to removal of the line, an infected hematoma which required an additional laparotomy, and a wound infection and subsequent wound dehiscence and evisceration which required additional surgery. In two

Table 3 Perioperative complications and additional procedures

\begin{tabular}{ll}
\hline Type of complication & $\begin{array}{l}\text { Number/surgical } \\
\text { procedures }^{\mathrm{a}}\end{array}$ \\
\hline Hemorrhage requiring transfusion & $33 / 0$ \\
Infection (CVL/sepsis/wound/hematoma/ascites) & $6 / 2$ \\
Biliary complications (bile leakage/cholestasis) & $9 / 8$ \\
Vascular complications & $2 / 2$ \\
Other & $2 / 1$
\end{tabular}

$C V L$ central venous line

${ }^{a}$ Number of patients receiving additional surgery due to complication

Table 4 Staging and postoperative outcomes after partial hepatectomy

\begin{tabular}{lll}
\hline Stage & $\begin{array}{l}\text { With complications } \\
(n=42) \\
\text { Number }\end{array}$ & $\begin{array}{l}\text { Without complications } \\
(n=31) \\
\text { Number }\end{array}$ \\
\hline PRETEXT 1 & 1 & 2 \\
PRETEXT 2 & 13 & 11 \\
PRETEXT 3 & $15\left(3^{\mathrm{a}}\right)$ & 6 \\
PRETEXT 4 & 3 & 4 \\
Unknown & $10\left(1^{\mathrm{a}}\right)$ & 8
\end{tabular}

PRETEXT pretreatment extent of disease

${ }^{\text {a }}$ Number of patients died of disease patients with infected ascites and clostridium enterocolitis, no additional surgery was needed.

Biliary complications Nine of seventy three $(12 \%)$ patients who underwent a partial hepatectomy experienced biliary complications. Eight patients developed a bile leakage and one patient developed cholestasis due to a biliary stricture. Eight of nine patients experiencing problems with biliary drainage needed one or more additional interventions (median 3; range 1-22 procedures) to resolve the biliary complication. These procedures included percutaneous biliary drainage of a biloma with or without leaving a drain in situ, endoscopic retrograde cholangiopancreatography (ERCP) with sphincterotomy, with or without biliary stenting, and percutaneous hepatic cholangiography (PTC) with biliary drainage and Roux-en-Y hepaticojejunostomy. In the remaining patient, problems resolved spontaneously.

Vascular complications Two (3\%) patients who underwent partial liver resection developed postoperative problems with hepatic blood flow. The first patient underwent a partial liver resection combined with a reconstruction of the caval- and left hepatic vein due to tumor ingrowth. Eventually, this patient developed the BuddChiari Syndrome with portal hypertension and liver cirrhosis. This resulted in multiple surgical procedures and eventually led to liver transplantation 9 years after the initial liver resection.

The second patient developed postoperative liver function problem due to thrombosis of the portal and left hepatic vein. Surgical revascularization and percutaneous thrombectomy were unsuccessful, and eventually, this patient received a liver transplant 7 days after the initial liver surgery with good outcome.

Other One patient with delayed gastric emptying needed a duodenal feeding tube that had to be placed under general anesthesia. In one patient, ECG abnormalities were reported during surgery, most likely due to an air embolism.

\section{Postoperative mortality}

No early deaths (within 30 days) were reported after partial hepatectomy.

However, one patient with pulmonary metastases died within 30 days after metastasectomy. This patient developed respiratory insufficiency due to persistent air leakage after extensive pulmonary surgery. Re-thoracotomy could not resolve the leakage.

\section{Recurrent disease}

Sixteen (17\%) of the ninety four patients eligible for surgery with curative intent presented with recurrent disease. In three patients, recurrent disease was diagnosed 


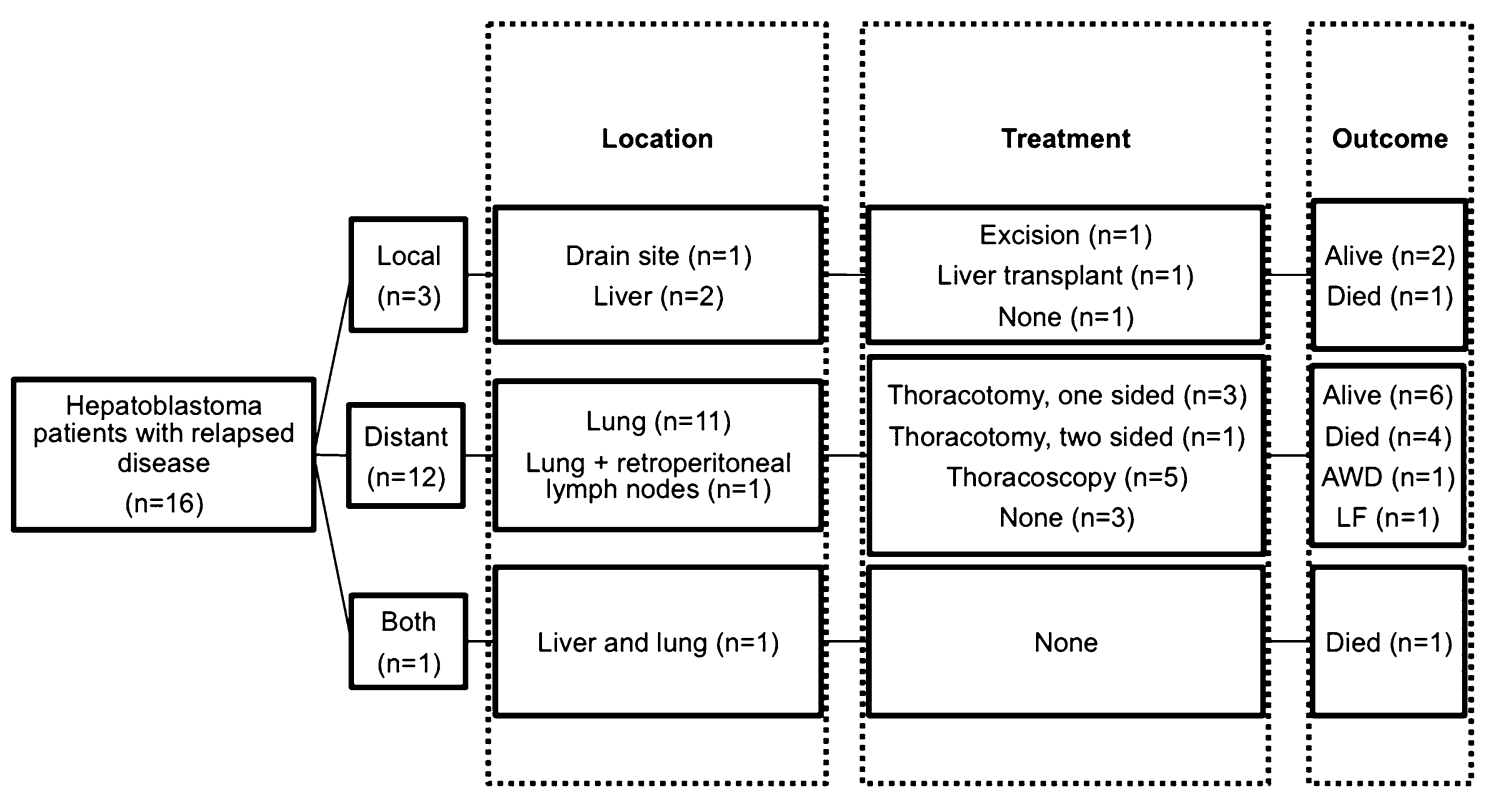

Fig. 3 Management and outcomes for hepatoblastoma patients with relapsed disease (1990-2013)

after primary total hepatectomy and transplantation, and in 13 patients, recurrent disease was diagnosed after partial liver resection. Details of management and outcomes of these 16 patients are shown in Fig. 3 .

\section{Local}

Two patients who underwent partial hepatectomy presented with recurrent disease involving the liver. One of these patients underwent rescue transplantation and the other patient died because of progressive disease.

In one patient, recurrent disease was found at the entry site of the intra-abdominal drain placed post operatively (drain site metastasis). The tumor was successfully removed.

\section{Distant}

Figure 3 shows that in 6 of 12 patients diagnosed with distant relapse, a second complete remission could be achieved after metastasectomy. At the time of analysis, one patient with pulmonary relapse was alive with recurrent disease. Three patients with pulmonary relapse and one patient with both pulmonary relapse and retroperitoneal lymph node metastases died because of progressive disease. One patient was lost to follow-up. Six patients with distant relapse were initially diagnosed with pulmonary metastases (Fig. 2).

\section{Both}

One patient developed both local and pulmonary recurrence. Further curative treatment was felt to be futile.

\section{Survival}

All but one death were related to progressive disease or due to relapse of the primary tumor. One patient was diagnosed with an intra-abdominal desmoid tumor and died. Figure 4 shows results for disease-specific survival for different groups. Overall, 5-year survival was $82 \% \quad(95 \%$ CI 74-89). In the group of 76 patients who had a partial hepatectomy, 5-year survival was $92 \%$ (95\% CI 86-98). Table 4 shows that among the five patients who eventually died after partial hepatectomy, four patients experienced one or more postoperative complications. Three of these patients were staged PRETEXT 3, and in two patients, staging was not documented. In one patient, there was no information about complications (therefore, not shown in Table 4). Of 18 patients who had primary transplantation, the 5-year survival was $77 \%$ (95\% CI 58-97) (Fig. 4).

\section{Discussion}

Partial hepatectomy for hepatoblastoma in children is a complex procedure, but essential for the cure of this disease. The results of this study show that partial hepatectomy in this group of patients is associated with high complication rates (58\%). However, a large proportion of these complications were considered minor and 5-year survival rates are good $(92 \%)$.

In $45 \%$ of the patients who underwent a partial liver resection, a blood transfusion was required. This corresponds with high rates $(57 \%)$ of red blood cell transfusion found by Tannuri et al. after hepatic resections in children 


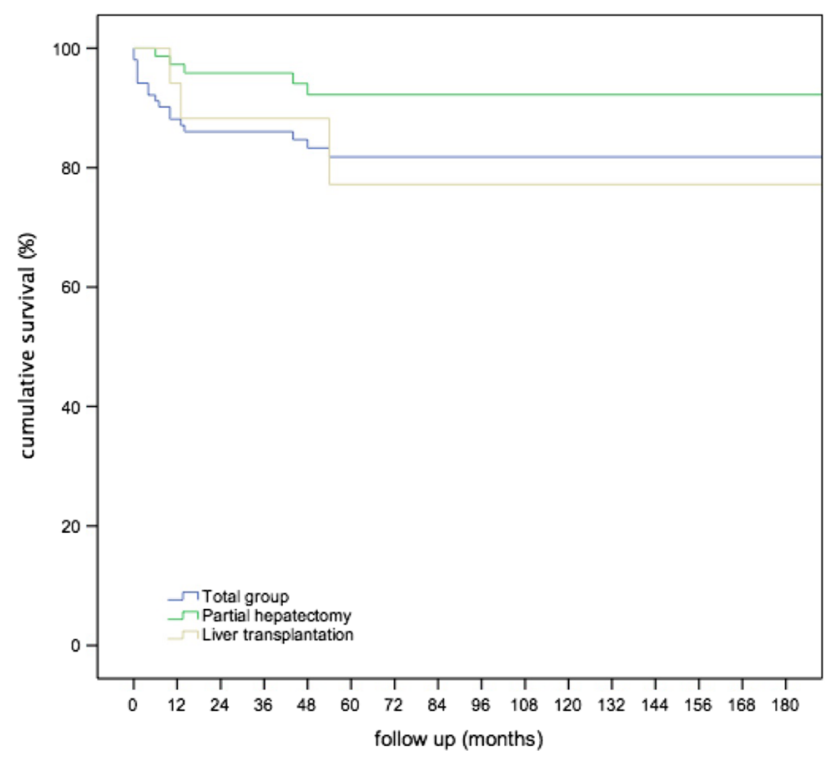

Fig. 4 5-year disease specific survival for patients with hepatoblastoma $(n=103)$, after partial hepatectomy $(n=76)$, after total hepatectomy and transplantation for patients with hepatoblastoma $(n=18)$ in the Netherlands (1990-2013)

with an estimated blood loss between 50-2000 mL (mean blood loss $280 \mathrm{~mL}$ ) [9]. Compared with adults, total blood volume is relatively small in pediatric patients. In this study, the median age at time of diagnosis was 15 months, which corresponds with mean circulating volume of 800-1000 mL. Young children do not tolerate blood loss as well as adults. Therefore, even a minor blood loss may necessitate transfusion. In adults, perioperative bleeding during partial hepatectomy is a common complication with higher incidence of liver failure and a shorter long-term survival $[21,22]$. There are no studies that support these findings in children. None of our patients needed a surgical re-intervention because of bleeding, but one needed CPR. The other patients could be treated with blood transfusion only. In our study, there was no association between blood loss and outcome after surgery, but the numbers of patients are relatively small.

Results of this study show that $12 \%$ of the patients developed biliary complications, and all but one needed one or more additional interventions to correct the underlying problem. Biliary complications after partial liver resection occur in 5-29\% of the children [11, 12, 17, 23]. If the injury is recognized during the surgical procedure, it can be repaired immediately. However, if biliary complications present after surgery, they are difficult to manage and often require additional surgery [12]. Results of a study by Steen et al. suggested that relatively high rates of biliary complications after partial liver resection in children might be associated with a high percentage of patients who received neoadjuvant chemotherapy [11]. The results of this study do not support this hypothesis, since the overall biliary complication rates were relatively low and all patients included in this study received neoadjuvant chemotherapy before undergoing partial liver resection. Furthermore, the results of the SIOPEL-1 study showed that neoadjuvant chemotherapy leads to downstaging of the tumor and results in a less extensive liver resection in $25 \%$ of the cases [1]. In addition, chemotherapy makes the tumor mass less prone to bleeding and better demarcated from the surrounding normal hepatic parenchyma, resulting in a safer and complete tumor resection [5]. Other possible explanations for developing more biliary complications, such as patient characteristics, and operative technique and surgeon experience should be further investigated.

Post-resection liver failure is a devastating complication that sometimes follows partial hepatic resection. In adults, the reported incidence of post-resection liver failure ranges between 0 and $32 \%$ [24, 25]. This percentage may be lower in children, since most children have no underlying parenchymal liver disease influencing liver function and usually show excellent hepatic regeneration and return of function after hepatic resection [26, 27]. However, there are no large studies to support this. In children, resection of $80 \%$ of the liver mass can be accepted after treatment with toxic agents [26]. To avoid long-term side effects of immunosuppressive therapy after transplantation, surgeons might extend criteria in favor of resection rather than transplantation in patients with extensive disease [28, 29]. Nonetheless, inadequate quantity or quality of residual liver mass remains a potential cause of liver failure and may require additional transplantation. Therefore, central review and surgical management at institutions experienced in both advanced pediatric hepatobiliary surgery and transplantation is essential for these children [28, 29]. In this study, two patients needed rescue transplantation after partial liver resection because of liver failure. In both patients, this was due to vascular complications after partial liver resection and not because of inadequate quantity or quality of the residual parenchyma. Both patients were still alive after 1 and 11 years of follow-up, respectively.

Once complete remission has been achieved, relapse is relatively rare $(<12 \%)$ [30] [31]. Prognostic factors for failure of the treatment of hepatoblastoma include the presence of metastasis, large tumor mass (high PRETEXT stage), age, small cell undifferentiated histology, and the $\alpha \mathrm{FP}$ level at presentation [31]. In most patients, recurrent disease presents with rising levels of $\alpha \mathrm{FP}$ [30]. Relapses can be local, metastatic, or combined. In this study, 16 patients $(17 \%)$ had recurrent disease; 3 patients presented with local recurrence, 12 with pulmonary relapse, and 1 patient with both local and distant relapse. Six out of twelve patients with pulmonary relapse were initially 
diagnosed with pulmonary metastasis and only one of these patients underwent metastasectomy. Therefore, chemotherapeutic failure rather than inadequate metastasectomy is a more likely cause of relapse. The place of more aggressive surgery in this group remains to be elucidated.

Once relapse occurs, a second remission can be achieved by treating the patient with chemotherapy, combined with surgical resection of the tumor mass. For pulmonary relapses, resection of the tumor by means of thoracotomy provides long-term cure in only $30 \%$ of the cases $[32,33]$. In this study, 6 of $12(50 \%)$ patients with pulmonary relapse survived (Fig. 3). In total, 6 (38\%) of 16 patients with relapsed disease survived. One patient was alive with recurrent disease and one patient was lost to follow-up.

Due to the retrospective character of this study, together with the long time period included in this study (24 years), retrievability of the data was challenging, especially for patients who underwent treatment in the pre digital period. For PRETEXT stage and tumour location, we restricted ourselves to patients' charts and we did not review old films. The challenges addressed above makes the case for prospective data collection even stronger and we advocate inclusion of surgical data in the SIOPEL analysis.

Centralization of these high-complex and low-volume procedures could potentially lead to further improvement of surgical technique and perioperative care and might have a beneficial effect on postoperative outcomes and survival as shown for other high complex (pediatric) surgical procedures [34-36]. Further research is necessary, since the effects of caseload and organization of care on outcomes for patients with hepatoblastoma have not yet been investigated.

\section{Conclusion}

In conclusion, this study shows that partial liver resection after chemotherapy in children with hepatoblastoma offers good chances of survival, even after pulmonary relapse. The results of this study show that partial hepatectomy in this group of patients is associated with high complication rates $(58 \%)$. However, a large proportion of these complications were considered minor. Four patients died after partial hepatectomy of local or distant relapse and this was never attributable to operative or postoperative complications.

Acknowledgments The authors acknowledge and thank Marco Schnater for providing data used in the previous research.

\section{Compliance with ethical standards}

Conflicts of interest The authors declare that they have no conflict of interest.

\section{References}

1. Schnater JM, Aronson DC, Plaschkes J et al (2002) Surgical view of the treatment of patients with hepatoblastoma: results from the first prospective trial of the International Society of Pediatric Oncology Liver Tumor Study Group. Cancer 94:1111-1120

2. Pritchard J, Brown J, Shafford E et al (2000) Cisplatin, doxorubicin, and delayed surgery for childhood hepatoblastoma: a successful approach-results of the first prospective study of the International Society of Pediatric Oncology. J Clin Oncol 18:3819-3828

3. Schnater JM, Kohler SE, Lamers WH et al (2003) Where do we stand with hepatoblastoma? A review. Cancer 98:668-678

4. Zsiros J, Brugieres L, Brock P et al (2013) Dose-dense cisplatinbased chemotherapy and surgery for children with high-risk hepatoblastoma (SIOPEL-4): a prospective, single-arm, feasibility study. Lancet Oncol 14:834-842

5. Aronson DC, Czauderna P, Maibach R et al (2014) The treatment of hepatoblastoma: its evolution and the current status as per the SIOPEL trials. J Indian Assoc Pediatr Surg 19:201-207

6. Dimick JB, Cowan JA Jr, Knol JA et al (2003) Hepatic resection in the United States: indications, outcomes, and hospital procedural volumes from a nationally representative database. Arch Surg 138:185-191

7. Towu E, Kiely E, Pierro A et al (2004) Outcome and complications after resection of hepatoblastoma. J Pediatr Surg 39:199-202 (discussion 199-202)

8. Pham TH, Iqbal CW, Grams JM et al (2007) Outcomes of primary liver cancer in children: an appraisal of experience. J Pediatr Surg 42:834-839

9. Tannuri AC, Tannuri U, Gibelli NE et al (2009) Surgical treatment of hepatic tumors in children: lessons learned from liver transplantation. J Pediatr Surg 44:2083-2087

10. Malek MM, Shah SR, Atri P et al (2010) Review of outcomes of primary liver cancers in children: our institutional experience with resection and transplantation. Surgery 148:778-782 (discussion 782-774)

11. Steen MW, Bakx R, Tabbers MM et al (2013) Endoscopic management of biliary complications after partial liver resection in children. J Pediatr Surg 48:418-424

12. Becker K, Furch C, Schmid I et al (2015) Impact of postoperative complications on overall survival of patients with hepatoblastoma. Pediatr Blood Cancer 62(1):24-28

13. Czauderna P, von Schweinitz D (2011) Surgical treatment. In: Zimmermann A, Perilongo G (eds) Pediatric Liver Tumors. Springer, Berlin

14. Perilongo G, Shafford E, Maibach R et al (2004) Risk-adapted treatment for childhood hepatoblastoma. final report of the second study of the International Society of Paediatric OncologySIOPEL 2. Eur J Cancer 40:411-421

15. Zsiros J, Maibach R, Shafford E et al (2010) Successful treatment of childhood high-risk hepatoblastoma with dose-intensive multiagent chemotherapy and surgery: final results of the SIOPEL3HR study. J Clin Oncol 28:2584-2590

16. Perilongo G, Maibach R, Shafford E et al (2009) Cisplatin versus cisplatin plus doxorubicin for standard-risk hepatoblastoma. N Engl J Med 361:1662-1670 
17. Schnater JM, Kuijper CF, Zsiros J et al (2005) Pre-operative diagnostic biopsy and surgery in paediatric liver tumours-the Amsterdam experience. Eur J Surg Oncol 31:1160-1165

18. Roebuck DJ, Aronson D, Clapuyt P et al (2007) 2005 PRETEXT: a revised staging system for primary malignant liver tumours of childhood developed by the SIOPEL group. Pediatr Radiol 37:123-132 (quiz 249-150)

19. Petty JKKF (2006) Liver resections. Hodder-Arnold Publishers, London

20. Otte JB, Pritchard J, Aronson DC et al (2004) Liver transplantation for hepatoblastoma: results from the International Society of Pediatric Oncology (SIOP) study SIOPEL-1 and review of the world experience. Pediatr Blood Cancer 42:74-83

21. Katz SC, Shia J, Liau KH et al (2009) Operative blood loss independently predicts recurrence and survival after resection of hepatocellular carcinoma. Ann Surg 249:617-623

22. Romano F, Garancini M, Uggeri F et al (2012) Bleeding in hepatic surgery: sorting through methods to prevent it. HPB Surg 2012:169351

23. Howat JM (1971) Major hepatic resections in infancy and childhood. Gut 12:212-217

24. van den Broek MA, Olde Damink SW, Dejong CH et al (2008) Liver failure after partial hepatic resection: definition, pathophysiology, risk factors and treatment. Liver Int 28:767-780

25. Hammond JS, Guha IN, Beckingham IJ et al (2011) Prediction, prevention and management of postresection liver failure. $\mathrm{Br} \mathbf{J}$ Surg 98:1188-1200

26. Wheatley JM, Rosenfield NS, Berger L et al (1996) Liver regeneration in children after major hepatectomy for malignancy-evaluation using a computer-aided technique of volume measurement. J Surg Res 61:183-189
27. Shamberger RC, Leichtner AM, Jonas MM et al (1996) Longterm hepatic regeneration and function in infants and children following liver resection. J Am Coll Surg 182:515-519

28. Lautz TB, Ben-Ami T, Tantemsapya N et al (2011) Successful nontransplant resection of POST-TEXT III and IV hepatoblastoma. Cancer 117:1976-1983

29. Fuchs J, Cavdar S, Blumenstock G, et al (2016) POST-TEXT III and IV hepatoblastoma: extended hepatic resection avoids liver transplantation in selected cases. Ann Surg (Epub head of print)

30. Semeraro M, Branchereau S, Maibach R et al (2013) Relapses in hepatoblastoma patients: clinical characteristics and outcomeexperience of the International Childhood Liver Tumour Strategy Group (SIOPEL). Eur J Cancer 49:915-922

31. Maibach R, Roebuck D, Brugieres L et al (2012) Prognostic stratification for children with hepatoblastoma: the SIOPEL experience. Eur J Cancer 48:1543-1549

32. Meyers RL, Czauderna P, Otte JB (2012) Surgical treatment of hepatoblastoma. Pediatr Blood Cancer 59:800-808

33. Meyers RL, Katzenstein HM, Krailo M et al (2007) Surgical resection of pulmonary metastatic lesions in children with hepatoblastoma. J Pediatr Surg 42:2050-2056

34. Lampela H, Ritvanen A, Kosola S et al (2012) National centralization of biliary atresia care to an assigned multidisciplinary team provides high-quality outcomes. Scand J Gastroenterol 47:99-107

35. Davenport M, Ong E, Sharif K et al (2011) Biliary atresia in England and Wales: results of centralization and new benchmark. J Pediatr Surg 46:1689-1694

36. Birkmeyer JD, Siewers AE, Finlayson EV et al (2002) Hospital volume and surgical mortality in the United States. N Engl J Med 346:1128-1137 